complete, and which aim only at giving a general idea of the nature of radium emanation therapy. I believe one need not be too much of an optimist to be able to endorse von Noorden's declaration that radium emanation therapy may now be justly regarded as belonging to the solid stock of medical therapeutic measures.

Bad Kreuznach.

\section{THE USE OF TUBERCULIN IN DIAGNOSIS AND TREATMENT. ${ }^{1}$}

\section{By J. F. HALLS Dally, M.A., M.D. Cantab.,} M.R.C.P. LOND.,

PHYSICIAN, AND PHYSICTAN IN CHARGE OF THE SPECIAL DEPARTMENT FOR TUBERCULOSIS, TO THE ST. MARYLEBONE GENERAL DISPENSARY; SENIOR ASSISTANT PHYSICIAN TO THE NATIONAL HOSPITAL FOR DISEASES OF 'THE HEAR'T.

Tuberoulin in diagnosis. - The specific tuberculin tests are four in number : (1) the ocular reaction of Wolff-Eisner and Calmette; (2) the cutaneous reaction of von Pirquet; (3) the percutaneous reaction of Moro; and (4) the subcutaneous reaction of Koch. All the above tests have their appropriate individual uses. They depend on the existence of that " paradox reaction" termed anaphylaxis.

1. Calmette's ocular reaction, described in 1307 , has since then attracted considerable attention. Many precautions are necessary in its application, and, even so, the test is not without a certain measure of risk to the eye, so that both for this reason and because, moreover, it is not as sensitive as the other three tests I have mentioned, it has now largely fallen into disuse in general medicine.

2. Von Pirquet's cutaneous reaction. The chief value of this reaction appears to be in the case of children under 5 years of age, where a positive reaction may be taken to indicate the presence of definite tuberculosis. In adolescents and adults a positive result must be reviewed in conjunction with all other available data before an opinion be given.

3. Moro's percutaneous reaction is a modified von Pirquet's reaction, and is readily performed by rubbing into a small portion of the skin an ointment of equal parts of old tuberculin and anhydrous lanolin. Occurrence of a localised dermatitis denotes a positive reaction. The ease and safety of this test are its chief advantages.

4. Koch's subcutaneous test is necessarily preceded by the patient's temperature being recorded three-hourly for at least three days before the injection, since this test should only be applied if the temperature is below $99^{\circ} \mathrm{F}$. For adults the first test dose consists of 0.001 c.c. old tuberculin (O.T). If no reaction ensues 0.005 c.c. O.T. is given in three days, and if still negative 0.01 c.c. O.T. at a like interval of time. In the continued absence of reactions one may say that no tuberculosis is present. For children over about 10 years of age the diagnostic dose may often be equivalent to that for adults, but for a child of 5 years of age the initial dose should not exceed 0.0005 c.c. O.T. and the next subsequent dose 0.0025 c.c. O.T.

The complete and typical reaction which may be expected, if tuberculosis is present, is in nature tripartite-viz., focal, local, and general, these manifestations being given in the order of importance. The focal reaction is essentially specific and produces hyperæmia and inflammatory swelling around the tuberculous focus, this in the lung being evidenced by the presence of new or increased moist crepitations in the affected patch, and appearance or increase of tubercle bacilli and elastic fibres in the sputum, which may be, and often is, itself increased in quantity. I regard the focal reaction as by far the most definite and conclusive of the three. Next comes the local reaction at the point of injection, which gives similar clinical appearances (apart from the papula) to the von Pirquet reaction. The general reaction is shown by fever and consequent malaise, sc. A rise of $10 \mathrm{~F}$. or more may be regarded as positive, and this comes on about 8 to 12 hours after the injection, appearing usually on the temperature chart as a peak with sharp elevation and decline, the downward slope at times being interrupted by a second rise

1 Abstract of a paper read at a mecting of the Chelsea Clinical Society on April 15th, 1913 . usually of lower, but occasionally of higher, level than the first.

Of all the diagnostic tests the subcutaneous one is, in my experience, the most reliable. If no tuberculosis is present tuberculin has no ill-effects. This fact alone encourages one to use tuberculin at the earliest moment to pick out cases suitable for sanatorium and tuberculin treatment from those for which these remedial methods are either inadvisable or unnecessary.

Tuberculin in treatment. - In the brief space at my disposal I cannot hope to do more than touch upon a few salient points based upon clinical observation in my tuberculosis department. The preparations of tuberculin are many and various. I find that some patients are more sensitive to one form of tuberculin and less sensitive to another, so that if intolerance of one kind of tuberculin is manifested it is wise to make a change. The main point to remember is that, no matter what preparation is used, sufficient time must be allowed for proper spacing of doses, and that it is never good policy to attempt to proceed too rapidly. If the remedy is to be beneficial, I believe that throughout one should try to avoid all but slight reactions, the only exception being in early stages of the weak preparations, where hypersensitiveness can be overcome by cautious increase of dosage at comparatively short intervals. This has the effect of inducing tolerance to the successive stronger doses, whereas repetition of the same or smaller doses at longer intervals appears to produce, and, if present, to intensify, that very condition of extreme sensitiveness which one is so anxious to avoid. Hence under such conditions one's motto must be, "Vestigia nulla retrorsum." Should, however, such procedure fail, the only course is to suspend the treatment for some weeks.

As to the scale of dosage which should be adopted there is no hard-and-fast rule. It is impossible briefly to give a set scale to which one should work, for not only has one to deal with each individual patient as a separate problem, bot also to be guided by the ever-varying factors of the patient's sensitiveness in particular and of his state in general. In my own practice I have abandoned other methods for the intensive system of dosage. In the treatment of pulmonary tuberculosis I begin with small doses of a weak bovine preparation-e.g., 1/10000 c.c. or less of P.T.O. - and working through increasing doses of this to bovine old tuberculin (P.T.). I complete the treatment in all cases where necessary by a course of human tuberculin, usually 0. T. or albumose-free old tuberculin (T.A.F.), finishing with doses of 1 c.c. or even more at longer intervals. In the earlier stages of treatment my custom is to inject every three or four days, and when larger doses of the strong preparations are reached, once a week.

Since the administrator cannot, with any accuracy, check the number of milligrammes of solid active substance con tained in the particular preparation used, I invariably record dosage in terms of cubic centimetres, for one can always measure exactly the amount of fluid that one injects. Tuberculin, when diluted, soon becomes inert, so that fresh dilutions should be made at least once a week. I nearly always make the dilution with freshly prepared saline solution containing 0.5 per cent. phenol at the time of administration. This procedure takes a little longer, but one thus avoids severe reactions consequent upon a potent dose following one which has been inactive.

On these lines immunisation can usually be attained within from 4 to 12 months, and various statistics show that such immunisation is capable of persisting over a period of several years.

In cases which are doing well the dosage can be increased more rapidly, so that the total time of immunisation is thus shortened. In advanced cases tuberculin does good at times in checking the rapid onward march of the disease, and the temperature may be brought to normal with apparent amelioration of physical signs to percussion and anseultation. Other cases, again, do badly. X ray examination in both these classes not infrequently reveals increase in shadow in the lungs, but in a case doing well in other respects it would be hard to say that such increased opacity represents extension of disease, since augmentation of visible shadow may quite well be due to attempt at repair by fibrosis. Should the disease progress notwithstanding cautious injections, tuberculin should be discontinued.

Lower Seymour street, W. 